\title{
Pancreatic cancer tissue banks: where are we heading?
}

\author{
“...for rapid advancement in pancreatic cancer research, national \\ endeavors such as the Pancreatic Cancer Research Fund Tissue Bank are \\ urgently needed.”
}

\begin{abstract}
Vickna Balarajah',2, Archana Ambily', Abu Z Dayem Ullah', Ahmet Imrali', Thomas Dowe', Bilal Al-Sarireh ${ }^{3}$, Mohammed Abu Hilal ${ }^{4}$, Brian R Davidson ${ }^{5}$, Zahir Soonawalla ${ }^{6}$, Matthew Metcalfe ${ }^{7}$, Jo-Anne Chin Aleong ${ }^{2}$, Claude Chelala' \& Hemant M Kocher ${ }^{* 1,2}$
\end{abstract}

First draft submitted: 21 May 2016; Accepted for publication: 8 August 2016; Published online: 19 August 2016

Pancreatic cancer is the fourth most common cause of cancer death in the UK [1]. In the USA, this is predicted to become the second most common cause of cancer death by 2030, overtaking deaths from breast and prostate cancer [2]. Worldwide, in 2012, the incidence of pancreatic cancer was 337,872 , with mortality figures of 330,391 that year, proving how lethal this cancer is [3]. In the UK alone, there were 9408 new diagnoses of pancreatic cancer in 2012 with an almost equal number of deaths [4].

Unfortunately, the mortality rate due to pancreatic cancer remains unchanged over the last 50 years worldwide [3]. Such statistics are largely due to vague or delayed presentation of the disease, which is partially a result of the pancreas being located deep in the abdomen. Despite modern advances in medical imaging, improvements in operative techniques and molecular understanding of the disease pathophysiology, the overall survival rate is still very poor. Often the disease is spread outside the organ or locally advanced at the time of diagnosis, thereby preventing curative treatments or therapies [1]. Operative options are available to less than $15 \%$ of patients; these depend on the site of the cancer and can either lead to a pancreaticoduodenectomy or a distal pancreatectomy [5]. Both surgical operations are, nevertheless, a huge undertaking for the patient with quoted procedural mortality of up to $2-11 \%$ and operative morbidity of up to $36-41 \%$ [6]. Post-surgical resection median survival is often described as 24 months, and 5-year survival is less than $25 \%$ [7]. On the other hand, locally advanced pancreatic cancer patients have a median survival of 6-10 months [8]. The estimated survival of a patient with untreated metastatic disease, the commonest stage of the disease at diagnosis, is $3-5$ months [1]. Those survival figures marginally improve with palliative

\section{KEYWORDS}

- biomarker • biorepository

- database $\bullet$ genomics $\bullet$ treatment

'Barts Cancer Institute - a Cancer Research UK Centre of Excellence, Queen Mary University of London, London, UK 


\author{
“...Pancreatic Cancer \\ Research Fund Tissue Bank \\ represents an effort to \\ embed an accepted \\ system of routine \\ biobanking at all levels of \\ public and private \\ healthcare systems for \\ pancreatic cancer.”
}

chemotherapy. The key to improving patient outcomes and survival is research into earlier detection of this highly complex and heterogeneous disease, which requires novel, tailored treatment options for each patient [9].

The pancreatic cancer research community, however, is small and insufficiently funded in comparison to research efforts in other cancers [10]. Researchers tend to develop bespoke collections of samples that may be unusable beyond the breadth and scope of their ethical approval and storage conditions. Sample collection protocols vary widely, thus limiting scope for collaboration with other research groups or extended sample usage. Such issues are globally acknowledged [10,11]. Examples of existing regional cancer tissue banks include Garvan Institute (Australia), Wales Cancer Bank (Wales) and Johns Hopkins (USA). Similarly, examples of single institute pancreas cancer tissue banks are those in Liverpool (UK); MD Anderson (TX, USA); UCLA Pancreas (CA, USA) and Barts Pancreas Tissue Bank (UK). In recognition of these issues, the All Party Parliamentary Group on Pancreatic Cancer in the UK recommended a focused approach and collaborative effort to encourage pancreatic cancer research in the country [12]. Among the many recommendations for advancing research and treatments on pancreatic cancer, the acknowledgement for research tissue banks were particularly significant [12].

The Pancreatic Cancer Research Fund Tissue Bank (PCRFTB) [13] aims to address these issues by developing a nationally (and, eventually, internationally) accessible repository of samples, collected under harmonized standard operating procedures. Biobanking has had an enormous impact in driving individual-tailored treatment regimens and healthcare [12]. Therefore, PCRFTB represents an effort to embed an accepted system of routine biobanking at all levels of public and private healthcare systems for pancreatic cancer. The aim is to develop a world-class clinically annotated bio-sample repository from patients with pancreatic diseases and cancer, which will act as a unique and vital resource for researchers investigating pancreatic cancer [13]. It is a dedicated research collaboration between clinicians, scientists, patients and their relatives. The PCRFTB will enable clinicians and scientists to work together to coordinate clinical and laboratory studies across the country [13]. The PCRFTB will target and accelerate synchronized pancreatic cancer research, avoiding common difficulties and pitfalls, such as local and fragmented tissue collections and research endeavors.

Patients will be encouraged to donate samples as early as possible along the treatment pathway, thus offering an opportunity to study disease development. Clinical information is gathered at the point of consent and the tissue bank personnel will be responsible for updating the clinical information as the treatment evolves. The option to consent to additional sample donations at multiple follow-up visits will ultimately create a catalog of longitudinal samples, potentially contributing to the identification of surveillance biomarkers. For example, patients who would potentially undergo surgery may have the opportunity to donate blood, urine, saliva as well as surplus tissue specimens beyond the requirement for staging and diagnosis. Patients upon entering the diagnostic pathway (endoscopy) or those undergoing systemic therapy will be offered to donate various types of bodily fluids to the Tissue Bank. By including non-cancer as well as nonoperable donors the research prospects will have an extensive scope with appropriate controls for biomarker development for diagnostic, predictive and prognostic applications.

All clinical samples and data will be compliant to uniform set of standard operating procedures. This will be recorded in a database supported by a secure web-based infrastructure. The donor identity will be protected by encrypting the person-identifiable data as well as using linked anonymized samples - in which a code links the sample to its donor - for research use. The rich clinical annotation will be available for mining clinical features of the disease in association with the research questions being asked, thus making research clinically applicable in a short time frame.

The PCRFTB aims to provide access to well-annotated samples and maximize the use of these tissues by implementing a data-return policy [13]. This ensures that any data generated using tissues obtained from the biobank are returned and made available to other researchers. Central to achieving this objective, a publicly available bioinformatics portal will be developed to provide data integration in two distinct ways. First, it will function as an online data-mining resource, permitting users to examine and collate pancreatic data from a single portal. Second, any data returned to the Tissue Bank will be incorporated into this bioinformatics repository, thus permitting researchers to mine the 
ever-increasing information associated with the tissues which are available for research.

We will build on the Pancreatic Expression Database [14], the leading repository for mining and integrating pancreatic literature data, for achieving the first aim. Ideal for downstream analysis, this unique tool offers pancreatic researchers the opportunity for broad and specific interrogation of over 280,000 measurements derived from a multitude of high-throughput profiles making it possible to access and combine data from a broad range of data types, experimental conditions, specimens and platforms for their own research. This enables identification of diagnostic and therapeutic targets relevant to researchers own experiments, cross-platform meta-analysis, thus hastening the development of novel diagnostic tools.

The seamless interoperability between the clinical database and bioinformatics portal will allow researchers to examine published findings prior to applying for tissues. In future, each PCRFTB sample will be linked to all the associated experimental findings relating to that sample or donor. This ensures that researchers will have access to the entire data catalog for every sample of interest, thus optimizing data sharing opportunities while minimizing duplication of effort.

The biorepository of the PCRFTB also aims to expand its activities towards extracting and storing DNA and RNA for future analysis with the prospect of deriving pancreatic cancer-specific cell lines from cancer as well as stroma. This would reduce, refine and replace experimentation in smaller animals following the $3 \mathrm{Rs}$ principle [15]. Indefinite storage of samples will enable future research with newer technologies. PCRFTB aims to develop cutting-edge technologies in cell and molecular biology, genomics, biomarker analysis, bioinformatics and ntegrating big-data analysis.

In conclusion for rapid advancement in pancreatic cancer research, national endeavors such as the PCRFTB are urgently needed.

\section{Acknowledgements}

The authors thank all patients and relatives for donating the samples and providing the clinical information to the Tissue Bank.

\section{Financial \& competing interests disclosure}

The authors thank Pancreatic Cancer Research Fund for funding the National Pancreas Registry and Tissue Bank also known as Pancreatic Cancer Research Fund Tissue Bank. The authors have no other relevant affliations or financial involvement with any organization or entity with a financial interest in or financial conflict with the subject matter or materials discussed in the manuscript apart from those disclosed.

No writing assistance was utilized in the production of this manuscript.

\section{References}

1 Hariharan D, Saied A, Kocher HM. Analysis of mortality rates for gallbladder cancer across the world. HPB (Oxford) 10(5), 327-331 (2008).

2 Rahib L, Smith BD, Aizenberg R, Rosenzweig AB, Fleshman JM, Matrisian LM. Projecting cancer incidence and deaths to 2030: the unexpected burden of thyroid, liver, and pancreas cancers in the United States. Cancer Res. 74(11), 2913-2921 (2014).

3 Kruger S, Haas M, Ormanns S et al. Translational research in pancreatic ductal adenocarcinoma: current evidence and future concepts. World J. Gastroenterol. 20(31), 10769-10777 (2014).

4 Pancreatic Cancer Statistics. www.cancerresearchuk.org
5 Williams NS, Christopher JKB, O'Connell PR (Eds). Bailey and Love's Short Practice of Surgery (25th Edition). A Hodder Arnold Publication, London, UK (2008).

6 Ho CK, Kleeff J, Friess H, Buchler MW. Complications of pancreatic surgery. $H P B$ (Oxford) 7(2), 99-108 (2005).

7 Kocher HM, Alrawashdeh W. Pancreatic cancer. BMJ Clin. Evid. 2010 (2010).

O'Sullivan A, Kocher HM. Pancreatic cancer. BMJ Clin. Evid. 2007 (2007).

9 Bailey P, Chang DK, Nones K et al. Genomic analyses identify molecular subtypes of pancreatic cancer. Nature 531(7592), 47-52 (2016). Consensus report of the national cancer institute clinical trials planning meeting on pancreas cancer treatment. J. Clin. Oncol. 27(33), 5660-5669 (2009).

11 Sjoquist KM, Chin VT, Chantrill LA et al. Personalising pancreas cancer treatment: when tissue is the issue. World J. Gastroenterol. 20(24), 7849-7863 (2014).

12 Pancreatic Cancer UK. www.pancreaticcancer.org.uk/about-us/

13 Pancreatic Cancer Research Fund Tissue Bank. www.pcrf.org.uk/pages/tissue-bank.html

14 Dayem Ullah AZ, Cutts RJ, Ghetia M et al. The pancreatic expression database: recent extensions and updates. Nucleic Acids Res. 42(Database issue), D944-D949 (2014).

15 The 3Rs. www.nc3rs.org.uk 